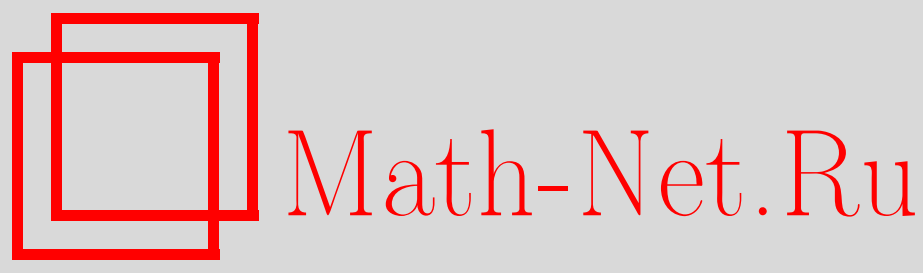

Р. В. Пальвелев, Рассеяние вихрей в абелевой модели Хиггса, ТМФ, 2008, том 156, номер 1, 77-91

DOI: https://doi.org/10.4213/tmf6231

Использование Общероссийского математического портала Math-Net.Ru подразумевает, что вы прочитали и согласны с пользовательским соглашением http://www . mathnet.ru/rus/agreement

Параметры загрузки:

IP: 34.227 .88 .159

26 апреля 2023 г., 05:58:38

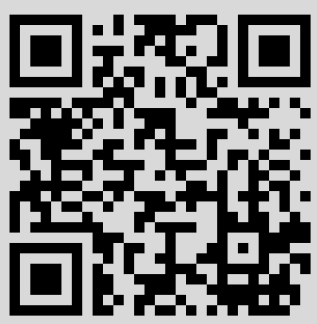




\section{РАССЕЯНИЕ ВИХРЕЙ В АБЕЛЕВОЙ МОДЕЛИ ХИГГСА}

Изучается рассеяние вихрей в абелевой $(2+1)$-мерной модели Хиггса. Показано, что в случае столкновения $N$ вихрей под равными углами их траектории после столкновения поворачиваются на угол $\pi / N$.

Ключевые слова: уравнения вихрей, уравнения Гинзбурга-Ландау, адиабатический предел, рассеяние вихрей.

В данной работе мы изучаем рассеяние вихрей в абелевой $(2+1)$-мерной модели Хиггса. Вихри, которые мы рассматриваем, - это решения вихревых уравнений, возникающих в теории сверхпроводимости. Они задаются гладкими парами $(A, \Phi)$, состоящими из (электромагнитного) калибровочного потенциала $A$ и скалярного поля Хиггса $\Phi$ на плоскости $\mathbb{C}$. $\mathrm{C}$ точностью до калибровочной эквивалентности вихревые решения параметризуются нулями поля Хиггса $\Phi$, так что пространство модулей $N$-вихревых решений можно отождествить с $\mathbb{C}^{N}$.

Динамика вихрей на $\mathbb{C}$ задается гиперболическим функционалом действия Гинзбурга-Ландау. Динамика $N$ вихрей приближенно описывается геодезическими в пространстве $\mathbb{C}^{N}$ в метрике, определяемой кинетической энергией модели. $\mathrm{K}$ сожалению, эту метрику не удается вычислить в явном виде. Однако в специальном случае симметричного столкновения $N$ вихрей мы можем показать, не используя явного вида метрики, что их траектории после столкновения поворачиваются на угол $\pi / N$. В частности, в случае двух вихрей их траектории после лобового столкновения поворачиваются на угол $\pi / 2$, т.е. происходит рассеяние под прямым углом. Последний результат был уже получен ранее в ряде работ (см. [1]-[4]).

\section{1. ВИХРЕВЫЕ РЕШЕНИЯ В АБЕЛЕВОЙ МОДЕЛИ ХИГГСА}

Двумерная абелева модель Хиггса задается следующим функционалом действия:

$$
V(\alpha, \Phi)=\frac{1}{2} \int_{\mathbb{R}^{2}}\left(\left|d_{\alpha} \Phi\right|^{2}+F_{12}^{2}+\frac{\lambda}{4}\left(|\Phi|^{2}-1\right)^{2}\right) d x d y
$$

где $\alpha=-i A_{1} d x-i A_{2} d y$ - калибровочный потенциал с гладкими вещественнозначными коэффициентами $A_{1}, A_{2}$ на $\mathbb{R}^{2}, \Phi=\Phi_{1}+i \Phi_{2}-$ поле Хиггса, задаваемое

* Московский государственный университет им. М. В. Ломоносова, Москва, Россия. E-mail: palvelev@front.ru 
гладкой комплекснозначной функцией на плоскости $\mathbb{R}^{2}, \lambda>0$ - константа. Через $F_{12}:=\partial_{1} A_{2}-\partial_{2} A_{1}$ мы обозначаем калибровочное поле, порождаемое потенциалом $\left(A_{1}, A_{2}\right)$. Здесь и далее $\partial_{1}:=\partial_{x}, \partial_{2}:=\partial_{y}$.

Функционал действия $V$ инвариантен относительно калибровочных преобразований следующего вида:

$$
\alpha \mapsto \tilde{\alpha}=\alpha-i d \chi, \quad \Phi \mapsto \widetilde{\Phi}=e^{i \chi} \Phi,
$$

где $\chi$ - гладкая вещественнозначная функция на $\mathbb{R}^{2}$.

Интегрируя по частям, мы можем переписать функционал действия $V$ в виде формулы Богомольного:

$$
\begin{aligned}
V=\frac{1}{2} & \int_{\mathbb{R}^{2}}\left\{\left(\left(\partial_{1} \Phi_{1}+A_{1} \Phi_{2}\right) \mp\left(\partial_{2} \Phi_{2}-A_{2} \Phi_{1}\right)\right)^{2}+\right. \\
& \left.+\left(\left(\partial_{2} \Phi_{1}+A_{2} \Phi_{2}\right) \pm\left(\partial_{1} \Phi_{2}-A_{1} \Phi_{1}\right)\right)^{2}+\left(F_{12} \pm \frac{1}{2}\left(|\Phi|^{2}-1\right)\right)^{2}\right\} d x d y \pm \\
& \pm \frac{1}{2} \int_{\mathbb{R}^{2}} F_{12} d x d y+\frac{\lambda-1}{4} \int_{\mathbb{R}^{2}}\left(|\Phi|^{2}-1\right)^{2} d x d y .
\end{aligned}
$$

Далее мы рассматриваем лишь критический случай $\lambda=1$ (см. [5]). Тогда правая часть последнего равенства есть сумма неотрицательных слагаемых и члена

$$
\frac{1}{2} \int_{\mathbb{R}^{2}} F_{12} d x d y,
$$

который пропорционален топологическому заряду или вихревому числу $N$ поля $(\alpha, \Phi)$. Вихревое число - это целое число, которое равно (см. [5])

$$
\frac{1}{2 \pi} \int_{\mathbb{R}^{2}} F_{12} d x d y=N,
$$

если выполнены следующие условия: $F_{12} \in L^{1}\left(\mathbb{R}^{2}\right),|\Phi| \rightarrow 1$ при $r:=\sqrt{x^{2}+y^{2}} \rightarrow$ $\infty$ и, кроме того, $\left|d_{\alpha} \Phi\right| \leqslant C / r^{1+\delta}$. Очевидно, $N$ не меняется при калибровочных преобразованиях.

Зафиксируем вихревое число $N$ и предположим, что $N \geqslant 0$. Тогда из формулы Богомольного следует, что $V(\alpha, \Phi) \geqslant \pi N$ и минимальное значение $V$ (равное $\pi N$ ) достигается на решениях системы уравнений

$$
\begin{gathered}
\partial_{1} \Phi_{1}+A_{1} \Phi_{2}=\partial_{2} \Phi_{2}-A_{2} \Phi_{1}, \\
\partial_{2} \Phi_{1}+A_{2} \Phi_{2}=-\partial_{1} \Phi_{2}+A_{1} \Phi_{1}, \\
F_{12}=-\frac{1}{2}\left(|\Phi|^{2}-1\right),
\end{gathered}
$$

называемых вихревыми уравнениями. Эти уравнения инвариантны относительно калибровочных преобразований.

Введем на плоскости комплексную координату $z=x+i y$ и обозначим, как обычно, $\partial:=\partial_{z}:=\left(\partial_{1}-i \partial_{2}\right) / 2$ и $\bar{\partial}:=\partial_{\bar{z}}:=\left(\partial_{1}+i \partial_{2}\right) / 2$. Также пусть $A:=\left(A_{1}-i A_{2}\right) / 2$. Тогда первые два из вихревых уравнений можно переписать в виде

$$
\bar{\partial} \Phi=i \bar{A} \Phi .
$$


Для полноты заметим, что в случае $N<0$ минимальное значение $V$, paвное $\pi|N|$, достигается на решениях похожей системы уравнений, называемых антивихревыми уравнениями. Любое решение $(\tilde{A}, \widetilde{\Phi})$ антивихревых уравнений можно получить из некоторого решения $(A, \Phi)$ вихревых уравнений по формулам $\tilde{A}(z)=-\bar{A}(-\bar{z})$, $\widetilde{\Phi}(z)=\Phi(-\bar{z})$. Поэтому мы ограничимся рассмотрением случая $N \geqslant 0$.

В [5] доказана следующая теорема существования и единственности.

Теорема 1 (Таубс). Пусть $N \geqslant 0, Z_{1}, Z_{2}, \ldots, Z_{N}$ - произвольные (не обязательно различные) точки на комплексной плоскости. Тогда существует такое решение $\left(A_{1}, A_{2}, \Phi\right)$ вихревых уравнений, что нули $\Phi$ совпадают с точками $Z_{1}, \ldots, Z_{N} u$

$$
\Phi(z, \bar{z}) \sim c_{j}\left(z-Z_{j}\right)^{n_{j}}
$$

в окрестности каждой из точек $Z_{j}$. Здесъ $n_{j}-$ кратность $Z_{j}$ в наборе $\left\{Z_{1}, \ldots, Z_{N}\right\}$, $c_{j}$ - ненулевая константа.

Для этого решения $|\Phi|$ экспоненииально стремится $\kappa$ единице при $|z| \rightarrow \infty, a$ $\left|\left(\partial_{1}-i A_{1}\right) \Phi\right| u\left|\left(\partial_{2}-i A_{2}\right) \Phi\right|$ экспоненциально убывают. Более точно,

$$
\left|d_{\alpha} \Phi\right| \leqslant C(1-|\Phi|)
$$

для некоторого $C>0$ и для любого $\delta>0$ существует $C(\delta)>0$ такое, ито

$$
1-|\Phi| \leqslant C(\delta) e^{-(1-\delta)|z|}
$$

Вихревое число этого решения равно N. Решение с указанными свойствами единственно с точностью до калибровочной эквивалентности.

Решение, существование которого устанавливается этой теоремой, называется $N$-вихревым решением.

Пространство модулей $N$-вихревых решений, обозначаемое через $\mathcal{M}_{N}$, - это множество классов $N$-вихревых решений с точностью до калибровочной эквивалентности. С помощью теоремы Таубса мы можем отождествить $\mathcal{M}_{N}$ с $N$-й симметрической степенью $S^{N} \mathbb{C}$, т.е. со множеством неупорядоченных наборов $N$ комплексных чисел (а именно, нулей $\Phi$ ). Эту симметрическую степень можно отождествить с $\mathbb{C}^{N}$, сопоставив каждому набору $\left\{Z_{1}, \ldots, Z_{N}\right\}$ полином $p(z)$ с нулями $Z_{1}, \ldots, Z_{N}$ и старшим коэффициентом 1:

$$
p(z)=\left(z-Z_{1}\right) \ldots\left(z-Z_{N}\right)=z^{N}+S_{1} z^{N-1}+\cdots+S_{N-1} z+S_{N} .
$$

\section{2. КАСАТЕЛЬНОЕ РАССЛОЕНИЕ $\mathcal{M}_{N}$ И КИНЕТИЧЕСКАЯ МЕТРИКА НА $\mathcal{M}_{N}$}

Касательное пространство к $\mathcal{M}_{N}$ в любой точке совпадает с $\mathbb{C}^{N}$. Однако, следуя [6], мы можем дать более детальное описание касательного расслоения $\mathcal{M}_{N}$. Это описание $T \mathcal{M}_{N}$ будет использовано при определении специальной кинетической метрики на $\mathcal{M}_{N}$. 
Для данной точки $\left\{Z_{1}, \ldots, Z_{N}\right\} \in \mathcal{M}_{N}$ мы рассмотрим определенное $N$-вихревое решение $\left(A_{1}, A_{2}, \Phi\right)$, которое сопоставляется точке $\left\{Z_{1}, \ldots, Z_{N}\right\}$ по теореме Таубса, причем калибровку зафиксируем следующим образом:

$$
\Phi(z)=\left(z-Z_{1}\right)\left(z-Z_{2}\right) \ldots\left(z-Z_{N}\right) f(z), \quad f(z)>0 .
$$

Теперь рассмотрим линеаризованные уравнения вихрей (в окрестности решения $\left.\left(A_{1}, A_{2}, \Phi\right)\right)$, которые имеют вид

$$
\begin{aligned}
\partial_{1} \varphi_{1}+a_{1} \Phi_{2}+A_{1} \varphi_{2} & =\partial_{2} \varphi_{2}-a_{2} \Phi_{1}-A_{2} \varphi_{1} \\
\partial_{2} \varphi_{1}+a_{2} \Phi_{2}+A_{2} \varphi_{2} & =-\partial_{1} \varphi_{2}+a_{1} \Phi_{1}+A_{1} \varphi_{1} \\
\partial_{1} a_{2}-\partial_{2} a_{1} & =-\Phi_{1} \varphi_{1}-\Phi_{2} \varphi_{2} .
\end{aligned}
$$

Здесь $\left(a_{1}, a_{2}, \varphi\right)$ - возмущение, $\varphi=\varphi_{1}+i \varphi_{2}$. Эти уравнения инвариантны относительно инфинитезимальных калибровочных преобразований вида

$$
a_{1} \mapsto \tilde{a}_{1}=a_{1}+\partial_{1} \chi, \quad a_{2} \mapsto \tilde{a}_{2}=a_{2}+\partial_{2} \chi, \quad \varphi \mapsto \tilde{\varphi}=\varphi+i \chi \Phi,
$$

где $\chi$ - вещественнозначная функция.

Рассмотрим пространство решений $\left(a_{1}, a_{2}, \varphi_{1}, \varphi_{2}\right)$ линеаризованных вихревых уравнений, принадлежащих пространству Соболева $\left(H^{1}\left(\mathbb{R}^{2} ; \mathbb{R}\right)\right)^{4}$. Заметим, что инфинитезимальные калибровочные преобразования с $\chi \in H^{2}\left(\mathbb{R}^{2}\right)$ переводят это пространство в себя. Следуя [6], мы зафиксируем инфинитезимальную калибровку, накладывая на решения $\left(a_{1}, a_{2}, \varphi_{1}, \varphi_{2}\right)$ линеаризованных уравнений вихрей следующее условие $L^{2}$-ортогональности:

$$
\int_{\mathbb{R}^{2}}\left(-\varphi_{1} \chi \Phi_{2}+\varphi_{2} \chi \Phi_{1}+a_{1} \partial_{1} \chi+a_{2} \partial_{2} \chi\right) d x d y=0 \quad \forall \chi \in H^{2}\left(\mathbb{R}^{2}\right) .
$$

Интегрируя по частям, получаем

$$
\int_{\mathbb{R}^{2}}\left(-\varphi_{1} \Phi_{2}+\varphi_{2} \Phi_{1}-\partial_{1} a_{1}-\partial_{2} a_{2}\right) \chi d x d y=0
$$

для любого $\chi \in H^{2}\left(\mathbb{R}^{2}\right)$, откуда вытекает, что

$$
\varphi_{1} \Phi_{2}-\varphi_{2} \Phi_{1}+\partial_{1} a_{1}+\partial_{2} a_{2}=0
$$

Это соотношение мы будет называть условием ортогональности.

Теперь введем линеаризованный вихревой оператор $\mathcal{D}_{(A, \Phi)}$, определяемый линеаризованными вихревыми уравнениями:

$$
\mathcal{D}_{(A, \Phi)}\left(a_{1}, a_{2}, \varphi\right)=\left(\begin{array}{c}
\partial_{1} \varphi_{1}+a_{1} \Phi_{2}+A_{1} \varphi_{2}-\partial_{2} \varphi_{2}+a_{2} \Phi_{1}+A_{2} \varphi_{1} \\
\partial_{2} \varphi_{1}+a_{2} \Phi_{2}+A_{2} \varphi_{2}+\partial_{1} \varphi_{2}-a_{1} \Phi_{1}-A_{1} \varphi_{1} \\
\partial_{1} a_{2}-\partial_{2} a_{1}+\Phi_{1} \varphi_{1}+\Phi_{2} \varphi_{2} \\
\varphi_{1} \Phi_{2}-\varphi_{2} \Phi_{1}+\partial_{1} a_{1}+\partial_{2} a_{2}
\end{array}\right)
$$

Это ограниченный линейный оператор, который отображает $\left(H^{1}\right)^{4}$ в $\left(L^{2}\right)^{4}$. Ядро $\mathcal{D}_{(A, \Phi)}$ имеет (вещественную) размерность $2 N$ (см. [6]). 
Мы будем отождествлять $\operatorname{ker} \mathcal{D}_{(A, \Phi)}$ с касательным пространством к $\mathcal{M}_{N}$ в точке, определяемой (согласно теореме Таубса) набором $\left\{Z_{1}, \ldots, Z_{N}\right\}$ нулей $\Phi$. Для этого рассмотрим симметрические функции $S_{1}, \ldots, S_{N}$ нулей $\Phi$ (равные коэффициентам полинома со старшим коэффициентом 1 , имеющего нули $\left.\left\{Z_{1}, \ldots, Z_{N}\right\}\right)$ как координаты на $\mathcal{M}_{N}$. Зафиксировав калибровку условием (4), мы можем рассматривать компоненты $\Phi, A_{1}, A_{2}$ вихревых решений как функции комплексных параметров $S_{1}, \ldots, S_{N}$. Решения линеаризованных уравнений будем строить, дифференцируя эти функции по параметрам.

Обозначим $S_{j, 1}:=\operatorname{Re} S_{j}, S_{j, 2}:=\operatorname{Im} S_{j}, j=1,2, \ldots, N$. Хотелось бы определить кинетическую метрику на $\mathcal{M}_{N}$ с помощью $L^{2}$-норм производных $\left(A_{1}, A_{2}, \Phi_{1}, \Phi_{2}\right)$ по переменным $S_{j, k}, j=1,2, \ldots, N, k=1,2$. K сожалению, эти производные могут не быть квадратично-интегрируемыми. Однако мы можем заменить $\left(A_{1}, A_{2}, \Phi_{1}, \Phi_{2}\right)$ на подходящие калибровочно-эквивалентные функции так, что их производные уже будут лежать в $L^{2}$. Другими словами, мы можем найти калибровочные множители $\chi_{j, k}$ так, чтобы функции

$$
n_{j, k}=\left(\frac{\partial A_{1}}{\partial S_{j, k}}+\partial_{1} \chi_{j, k} \frac{\partial A_{2}}{\partial S_{j, k}}+\partial_{2} \chi_{j, k} \frac{\partial \Phi_{1}}{\partial S_{j, k}}-\chi_{j, k} \Phi_{2} \frac{\partial \Phi_{2}}{\partial S_{j, k}}+\chi_{j, k} \Phi_{1}\right)
$$

лежали в $\left(H^{1}\right)^{4}$ и удовлетворяли условию ортогональности. Векторы $n_{j, k}$ при $j=$ $1,2, \ldots, n, k=1,2$, лежат в пространстве $\operatorname{ker} \mathcal{D}_{(A, \Phi)}$ и являются линейно независимыми. Поэтому они образуют базис в $\operatorname{ker} \mathcal{D}_{(A, \Phi)}$. Используя этот базис, мы можем отождествить $T_{(A, \Phi)} \mathcal{M}_{N}$ и $\operatorname{ker} \mathcal{D}_{(A, \Phi)}$.

Теперь мы можем определить кинетическую метрику $g$ на $\mathcal{M}_{N}$ с помощью $L^{2}$-скалярного произведения. А именно, для любых векторов $v_{1}, v_{2} \in T_{(A, \Phi)} \mathcal{M}_{N}=$ $\operatorname{ker} \mathcal{D}_{(A, \Phi)} \subset\left(H^{1}\right)^{4}$ положим $g\left(v_{1}, v_{2}\right):=\left(v_{1}, v_{2}\right)_{\left(L^{2}\right)^{4}}$.

Для того чтобы обосновать корректность определения метрики и ее гладкость, мы докажем следующую теорему.

Tеорема 2. Пусть $A_{1}\left(z ; S_{1}, \ldots, S_{N}\right), A_{2}\left(z ; S_{1}, \ldots, S_{N}\right), \Phi\left(z ; S_{1}, \ldots, S_{N}\right)$ - компоненты вихревого решения, которое имеет нули в корнях многочлена $z^{N}+$ $S_{1} z^{N-1}+\cdots+S_{N}$ и удовлетворяет калибровочному условию (4). Тогда:

1) $\Phi, A_{1}, A_{2}$ гладко зависят от $S_{1}, \ldots, S_{N}$ в том смысле, что при фиксированном $z_{0}$ функиия $\Phi\left(z_{0} ; S_{1}, \ldots, S_{N}\right)$ - гладкая функиия аргументов $S_{1}, \ldots, S_{N}$ и то же самое верно для $A_{1}, A_{2}$;

2) можно выбрать функции $\chi_{j, k}$ так, чтобъ векторы $n_{j, k}$, определяемые формулой (6), лежали в $\left(H^{1}\right)^{4}$ и удовлетворяли условию ортогональности;

3) отображсения $\left(S_{1}, \ldots, S_{N}\right) \mapsto n_{j, k}\left(S_{1}, \ldots, S_{N}\right)-$ гладкие отображения $\mathbb{C}^{N} \rightarrow$ $\left(L^{2}\right)^{4}$ со значениями в $\left(H^{1}\right)^{4}$;

4) векторы $n_{j, k}$ при $j=1,2, \ldots, N, k=1,2$ линейно независимы.

Доказательство этой теоремы будет дано в разделе 4.

СлеДСТВИЕ. Коэфбициенты кинетической метрики суть гладкие функции координат $S_{1}, \ldots, S_{N}$. 
ДокАЗАТЕЛЬство. Коэффициенты метрики определяются по формулам $g_{j, k ; l, m}=\left(n_{j, k}, n_{l, m}\right)_{\left(L^{2}\right)^{4}}$. Из п. 3 теоремы 2 следует, что они являются гладкими функциями координат $S_{1}, \ldots, S_{N}$.

ЗАмЕчАниЕ. Из симметрии задачи следует, что кинетическая метрика является инвариантной относительно одновременных сдвигов всех нулей, их поворотов, а также относительно замены всех нулей на комплексно-сопряженные, т.е. относительно преобразований вида $Z_{j} \mapsto Z_{j}+Z_{0}, Z_{j} \mapsto e^{i \varphi} Z_{j}$ и $Z_{j} \mapsto \bar{Z}_{j}$. Это обстоятельство будет использовано при доказательстве теоремы 3.

\section{3. ДИНАМИЧЕСКАЯ ЗАДАЧА}

Динамическая $(2+1)$-мерная модель Хиггса задается функционалом действия

$$
\begin{aligned}
\mathcal{S}(A, \Phi)=\frac{1}{2} & \int_{t_{1}}^{t_{2}} d t \int_{\mathbb{R}^{2}}\left\{\left(\left|\left(\partial_{0}-i A_{0}\right) \Phi\right|^{2}+F_{01}^{2}+F_{02}^{2}\right)-\right. \\
& \left.-\left(\left|\left(\partial_{1}-i A_{1}\right) \Phi\right|^{2}+\left|\left(\partial_{2}-i A_{2}\right) \Phi\right|^{2}+F_{12}^{2}+\frac{1}{4}\left(|\Phi|^{2}-1\right)^{2}\right)\right\} d x d y .
\end{aligned}
$$

Здесь $\Phi(t, x, y)$ - гладкая комплекснозначная функция, $A_{j}(t, x, y)$ - гладкие вещественнозначные функции, $j=0,1,2, F_{j k}:=\partial_{j} A_{k}-\partial_{k} A_{j}$. (Мы обозначаем $\partial_{0}=\partial_{t}$, $\partial_{1}=\partial_{x}, \partial_{2}=\partial_{y}$.)

Функционал действия можно представить в стандартной форме

$$
\mathcal{S}=\int(T-V) d t
$$

где потенциальная энергия $V$ задается формулой (1), а кинетическая энергия $T$ равна

$$
T=\frac{1}{2} \int_{\mathbb{R}^{2}}\left(\left|\left(\partial_{0}-i A_{0}\right) \Phi\right|^{2}+F_{01}^{2}+F_{02}^{2}\right) d x d y .
$$

Хотелось бы описать пространство модулей соответствующей динамической задачи $\delta \mathcal{S}(A, \Phi)=0$. Но в отличие от вихревых решений (которые являются статическими решениями нашей динамической задачи), получить явное описание этого пространства модулей затруднительно. Однако, следуя идее, выдвинутой в [6] и [7], мы можем рассмотреть геодезические на $\mathcal{M}_{N}$ в кинетической метрике как хорошие приближения к траекториям $N$ медленно движущихся вихрей. Будем использовать это приближение, чтобы описать рассеяние $N$ вихрей после их симметричного столкновения.

K сожалению, для кинетической метрики нет явной формулы. Однако мы будем использовать инвариантность этой метрики относительно вращений и комплексного сопряжения, чтобы получить качественное описание рассеяния. А именно, мы докажем, что после симметричного столкновения траектории вихрей поворачиваются на угол $\pi / N$.

Под симметричным столкновением $N$ вихрей мы подразумеваем их движение в одну точку вдоль таких лучей с началом в этой точке, что угол между любыми 
соседними лучами равен $2 \pi / N$. Иначе говоря, в каждый момент времени вихри находятся в вершинах правильного $N$-угольника с центром в точке, в которую они движутся. Поскольку задача инвариантна относительно сдвигов и поворотов плоскости, мы можем предположить, что $N$ вихрей движутся в начало координат вдоль лучей $\arg z=0, \arg z=2 \pi / N, \ldots, \arg z=2(N-1) \pi / N$ и одновременно сталкиваются в начале координат. Утверждается, что после столкновения они разлетаются по лучам $\arg z=\pi / N, \ldots, \arg z=(2(N-1)+1) \pi / N$ (они получаются из исходных лучей поворотом на угол $\pi / N)$.

В частности, лобовое столкновение двух вихрей приводит к повороту их траекторий на угол $\pi / 2$, так что в этом случае мы наблюдаем рассеяние под прямым углом. Последний результат уже был получен в ряде работ (см. [1]-[4]).

Мы докажем математический результат (теорема 3), а затем дадим его физическую интерпретацию.

Теорема 3. На пространстве модулей $\mathcal{M}_{N}$ с кинетической метрикой имеется геодезическая, которую можно параметризовать в симметрических координатах следуюшим образом ${ }^{1)}$ :

$$
S_{1}=\cdots=S_{N-1}=0, \quad S_{N}(s)=(-1)^{N} \lambda(s),
$$

где $\lambda(s)$ - гладкая вещественнозначная убывающая функция, $\lambda(s)$ принимает все вещественные значения и $\lambda(0)=0$.

ДокАзАтЕльство. В силу следствия из теоремы 2 мы можем пользоваться стандартными результатами о существовании и единственности геодезических для кинетической метрики. Рассмотрим геодезическую, проходящую через начало координат $S_{1}=\cdots=S_{N}=0$ и имеющую в этой точке касательный вектор $\vec{v}=$ $\left(0,0, \ldots, 0,(-1)^{N+1}\right)$. Покажем, что она имеет требуемый вид. В самом деле, пусть наша геодезическая параметризована: $S_{1}=S_{1}(s), \ldots, S_{N}=S_{N}(s)^{2}$.

Воспользуемся свойствами инвариантности метрики (см. замечание после следствия из теоремы 2). Поворот нулей $\Phi$ на угол $\varphi\left(Z_{j} \mapsto e^{i \varphi} Z_{j}\right)$ в симметрических координатах соответствует преобразованию вида

$$
S_{1}^{\prime}=e^{i \varphi} S_{1}, \quad S_{2}^{\prime}=e^{2 i \varphi} S_{2}, \quad \ldots, \quad S_{N}^{\prime}=e^{i N \varphi} S_{N} .
$$

Значит, поворот на угол $2 \pi / N$ оставляет на месте вектор $\vec{v}$, касательный к кривой $S_{1}(t)=\cdots=S_{N-1}(t)=0, S_{N}(t)=(-1)^{N+1} t$, которая инвариантна относительно этого поворота. Из этого следует, что поворот на угол $2 \pi / N$ переводит нашу геодезическую в геодезическую, которая также проходит через начало координат и имеет в этой точке тот же самый касательный вектор $\vec{v}$. Из единственности геодезической мы получаем, что наша геодезическая инвариантна относительно этого

\footnotetext{
1) Множитель $(-1)^{N}$ в формулировке и в доказательстве появляется потому, что $S_{N}=$ $\left(-Z_{1}\right)\left(-Z_{2}\right) \ldots\left(-Z_{N}\right)=(-1)^{N} Z_{1} Z_{2} \ldots Z_{N}$.

2) Здесь $s$ - натуральный параметр на геодезической. Будем считать, что началу координат соответствует значение параметра $s=0$. Интервал изменения параметра выбирается максимальным.
} 
поворота: $S_{1}(s)=e^{2 \pi / N} S_{1}(s), \ldots, S_{N-1}(s)=e^{2 \pi(N-1) / N} S_{N-1}(s)$, а из этого следует, что $S_{1}(s)=\cdots=S_{N-1}(s)=0$.

Теперь мы используем комплексное сопряжение. В симметрических координа$\operatorname{tax} S_{1}, \ldots, S_{N}$ оно соответствует отображению $S_{1}^{\prime}=\bar{S}_{1}, S_{2}^{\prime}=\bar{S}_{2}, \ldots, S_{N}^{\prime}=\bar{S}_{N}$. Это отображение также переводит вектор $\vec{v}$ в себя и потому оставляет нашу геодезическую на месте. Значит, функции $S_{N}(s)$ и $\lambda(s):=(-1)^{N} S_{N}(s)$ вещественные. Очевидно, что $\lambda(s)$ убывает и при $s=0$ меняет знак с положительного на отрицательный.

Поскольку в симметрических координатах метрика не имеет особенностей, то наша геодезическая продолжается в обе стороны неограниченно, т.е. $(-1)^{N} S_{N}$ при возрастании $s$ меняется от $+\infty$ до $-\infty$. (В [2] отмечено, что при $\min _{j \neq l}\left|Z_{j}-Z_{l}\right| \rightarrow \infty$ метрика стремится к плоской метрике вида const $\sum_{j=1}^{N} d Z_{j} d \bar{Z}_{j}$. Поэтому интервал изменения $s$ есть вся прямая: $-\infty<s<\infty$.)

Физически теорему 3 можно интерпретировать следующим образом. В координатах $\left(Z_{1}, \ldots, Z_{N}\right)$ наша геодезическая (гладкая в симметрических координатах) описывает следующее движение $N$ вихрей. При $\lambda(s)>0$ (т.е. $s<0$ ) они движутся по траекториям

$$
s \mapsto\left(\sqrt[N]{\lambda(s)}, \sqrt[N]{\lambda(s)} e^{2 \pi i / N}, \ldots, \sqrt[N]{\lambda(s)} e^{2 \pi i(N-1) / N}\right)
$$

а при $\lambda(s)<0$ (т.е. $s>0)$ - по траекториям, повернутым на угол $\pi / N$ :

$$
s \mapsto\left(\sqrt[N]{|\lambda(s)|} e^{\pi i / N}, \sqrt[N]{|\lambda(s)|} e^{3 \pi i / N}, \ldots, \sqrt[N]{|\lambda(s)|} e^{(2 N-1) \pi i / N}\right) .
$$

Другими словами, наша геодезическая задает процесс рассеяния, описанный выше: до столкновения $N$ вихрей движутся в начало координат по лучам $\arg z=0, \arg z=$ $2 \pi / N, \ldots, \arg z=2(N-1) \pi / N$, затем они сталкиваются в начале координат и разлетаются по лучам $\arg z=\pi / N, \ldots, \arg z=(2 N-1) \pi / N$. В частности, при $N=2$ имеются два вихря, которые движутся навстречу друг другу по вещественной оси, сталкиваются и разлетаются по мнимой оси, т.е. происходит рассеяние под прямым углом.

Важно отметить, что теорема 3 дает не только локальный результат (т.е. главные члены асимптотики функций, описывающих движение вихрей на малом интервале времени после столкновения), но и глобальный результат о рассеянии: мы получаем полное описание траекторий вихрей на всем интервале времени как после столкновения, так и до него. В частности, получается, что симметричное столкновение возможно лишь тогда, когда вихри с самого начала (из бесконечности) движутся вдоль лучей $\arg z=0, \arg z=2 \pi / N, \ldots, \arg z=2(N-1) \pi / N$.

В задаче о рассеянии вихрей наиболее изученным является случай $N=2$. В частности, Стюарт [6] доказал для этого случая корректность определения и гладкость метрики и, более того, обосновал применимость геодезического приближения. Ранее результат о рассеянии двух вихрей был получен в [1]-[3] (с разной степенью строгости). Общий же случай (рассеяние $N$ вихрей при симметричном столкновении) был описан на “физическом" уровне строгости в статьях [8] и [9]. 
Следствие из теоремы 2 позволяет получать локальные результаты о рассеянии в некоторых более общих случаях. Рассмотрим следующий пример. Пусть имеется $N>2$ вихрей, движение которых описывается геодезической вида $S_{1}=S_{1}(s), \ldots$, $S_{N}=S_{N}(s)$. Пусть два из этих вихрей в момент $s=0$ сталкиваются в нуле, а остальные в этот момент находятся в других точках (это значит, что многочлен $z^{N}+S_{1}(0) z^{N-1}+\cdots+S_{N}(0)$ имеет в точке $z=0$ нуль кратности два, или $\left.S_{N}(0)=S_{N-1}(0)=0, S_{N-2}(0) \neq 0\right)$. Лобовым столкновением таких двух вихрей мы будем называть ситуацию, когда эти вихри до столкновения движутся по траекториям вида $Z_{1}(s)=d(s)(1+o(1))$ и $Z_{2}(s)=-d(s)(1+o(1))\left(\right.$ при $\left.s \rightarrow 0^{-}\right)$, где $d(s)$ - положительная непрерывная убывающая функция, определенная при $s \leqslant 0$, и $d(0)=0$. Возникает вопрос: как будут двигаться эти два вихря после столкновения?

Заметим, что в описанной ситуации при малых по модулю значениях $s$ можно представить многочлен $z^{N}+S_{1}(s) z^{N-1}+\cdots+S_{N}(s)$ в виде произведения

$$
\left(z^{2}+a(s) z+b(s)\right)\left(z^{N-2}+\widetilde{S}_{1}(s) z^{N-3}+\cdots+\widetilde{S}_{N-2}(s)\right),
$$

где коэффициенты $a(s), b(s), \widetilde{S}_{k}(s)$ - гладкие функции, $a(0)=b(0)=0$ и $\widetilde{S}_{N-2}(0) \neq 0$, т.е. корни первого многочлена - положения двух сталкивающихся вихрей, а корни второго - всех остальных. Ясно, что $b(s)=-d^{2}(s)(1+o(1))$. Рассмотрим лишь случай, когда $b^{\prime}(0) \neq 0$ (т.е. $b(s)=b_{1} s+o(s)$ при $\left.s \rightarrow 0\right)$. Тогда $-d^{2}(s)=b_{1} s(1+o(1))$ при малых отрицательных значениях $s$, и поэтому $b_{1}>0$. Решая квадратное уравнение, получаем $Z_{1,2}=\left(-a(s) \pm \sqrt{a^{2}(s)-4 b(s)}\right) / 2$. Но $a^{2}(s)-4 b(s)=-4 b_{1} s+O\left(s^{2}\right)$, а значит, $\sqrt{a^{2}(s)-4 b(s)}= \pm 2 i \sqrt{b_{1}} \sqrt{s}(1+O(s))$ при $s>0$. Следовательно, $Z_{1,2}=$ $\pm i \sqrt{b_{1}} \sqrt{s}+O(s)$. Это означает, что, несмотря на присутствие остальных вихрей, локально опять происходит рассеяние под прямым углом.

Следует заметить, что любая геодезическая, выходящая из точки $\left(S_{1}, \ldots\right.$ $\left.\ldots, S_{N-2}, 0,0\right)$ с $S_{N-2} \neq 0$ в направлении вектора $\left(a_{1}, \ldots, a_{N}\right)$ с $a_{N} \neq 0$, описывает локальное лобовое столкновение двух вихрей в нуле, имеющее вышеуказанный вид, так как в формуле $(8) b(s)=\left(a_{N} s / \widetilde{S}_{N-2}(0)\right)+o(s)$, а значит, $b^{\prime}(0) \neq 0$. То, что мы считали эти два вихря сталкивающимися вдоль вещественной оси, несущественно в силу инвариантности метрики относительно поворотов.

\section{4. ДОКАЗАТЕЛЬСТВО ТЕОРЕМЫ 2}

Гладкая зависимость от $S_{k}$. Система вихревых уравнений может быть сведена к одному нелинейному эллиптическому уравнению [5]. Это делается следующим образом. Мы полагаем

$$
\Phi=e^{(u+i \theta) / 2}, \quad A=\frac{1}{2}(u-i \theta),
$$

где $\theta=2 \sum_{k=1}^{N} \arg \left(z-Z_{k}\right)$. Затем представляем $u$ в виде $u=u_{0}+v$, где

$$
u_{0}=-\sum_{k=1}^{N} \ln \left(1+\frac{\mu}{\left|z-Z_{k}\right|^{2}}\right)
$$


а $v$-решение уравнения

$$
\Delta v=e^{u_{0}} e^{v}-1+g,
$$

в котором

$$
g(z)=4 \sum_{k=1}^{N} \frac{\mu}{\left(\mu+\left|z-Z_{k}\right|^{2}\right)^{2}} .
$$

При этом мы требуем, чтобы $v$ удовлетворяла асимптотическому условию $v(z) \rightarrow 0$ при $|z| \rightarrow \infty$ (число $\mu>4 N$ - вспомогательный параметр, который в сумме $u_{0}+v$ сокращается). Уравнение (9) имеет единственное гладкое решение, которое лежит в пространстве Соболева $H^{2}\left(\mathbb{R}^{2}\right)$ (см. [5]).

Применим теперь эту процедуру к нашему решению, которое удовлетворяет условию (4):

$$
\Phi(z)=\left(z-Z_{1}\right) \ldots\left(z-Z_{N}\right) f(z), \quad f(z)>0 .
$$

Обозначим $p(z):=\left(z-Z_{1}\right) \ldots\left(z-Z_{N}\right)$, тогда $\Phi(z)=p(z) f(z)$.

Мы докажем, что единственное (при каждом значении параметров) решение $v=$ $v\left(Z_{1}, \ldots, Z_{N}\right)$ уравнения (9) гладко зависит от параметров $\left(S_{1}, \ldots, S_{N}\right)$, т.е. симметрических функций от нулей $\left(Z_{1}, \ldots, Z_{N}\right)$. Нам будет проще доказать это утверждение для функции $w:=2 \ln f+\ln \left(1+|p|^{2}\right)$, которая отличается от $v$ на известную функцию:

$$
w=v+\ln \left(1+|p|^{2}\right)-\sum_{k=1}^{N} \ln \left(\mu+\left|z-Z_{k}\right|^{2}\right) .
$$

Заметим, что

$$
\ln \left(1+|p|^{2}\right)-\sum_{k=1}^{N} \ln \left(\mu+\left|z-Z_{k}\right|^{2}\right) \in H^{2}\left(\mathbb{R}^{2}\right) .
$$

Для функции $w$ мы получаем следующее уравнение:

$$
\Delta w=\frac{|p|^{2}}{1+|p|^{2}} e^{w}-1+\Delta \ln \left(1+|p|^{2}\right)
$$

Отметим, что $w$ является решением (10) тогда и только тогда, когда

$$
v=w-\ln \left(1+|p|^{2}\right)+\sum_{k=1}^{N} \ln \left(\mu+\left|z-Z_{k}\right|^{2}\right)
$$

является решением (9).

Мы докажем, что $\left(S_{1}, \ldots, S_{N}\right) \mapsto w\left(S_{1}, \ldots, S_{N}\right)$ есть гладкое отображение $\mathbb{C}^{N} \rightarrow$ $H^{2}\left(\mathbb{R}^{2}\right)$, используя следующую форму теоремы о неявной функции на банаховом пространстве.

ТЕорема 4 (теорема о неявной функции). Пусть $X, Y, Z$ - банаховы пространства, $W \subset X \times Y$ - окрестность точки $\left(x_{0}, y_{0}\right), \Psi: W \rightarrow Z$ - отображение класса $C^{1}(W)$ и $\Psi\left(x_{0}, y_{0}\right)=0$. Предположим, что существует ограниченный обратный оператор $\left(\Psi_{y}^{\prime}\left(x_{0}, y_{0}\right)\right)^{-1}: Z \rightarrow Y$. 
Тогда найдутся шар $B\left(x_{0}, \delta\right) \subset X$ и $C^{1}$-гладкое отображение $\phi: B\left(x_{0}, \delta\right) \rightarrow Y$ такие, что:

а) $\phi\left(x_{0}\right)=y_{0}$;

б) $\Psi(x, \phi(x)) \equiv 0$;

в) существует шар $B\left(y_{0}, \varepsilon\right) \subset Y$ такой, что $\phi(x)$ - единственное решение уравнения $\Psi(x, y)=0$ относительно $y$, лежащее в этом шаре.

Кроме того, если $\Psi \in C^{r}, r \in \mathbb{N}$, то и $\phi \in C^{r}\left(B\left(x_{0}, \delta\right)\right)$. (Значит, если $\Psi \in C^{\infty}$, mo $u \phi \in C^{\infty}$.)

Применим эту теорему к отображению $F: H^{2}\left(\mathbb{R}^{2}\right) \times \mathbb{C}^{N} \rightarrow L^{2}$, которое задается формулой

$$
F(v ; S)=-\Delta v+\frac{|p(S)|^{2}}{1+|p(S)|^{2}} e^{v}-1+\Delta \ln \left(1+|p(S)|^{2}\right)
$$

где $S:=\left(S_{1}, \ldots, S_{N}\right) \in \mathbb{C}^{N}$ (здесь $v$ - не обязательно решение (9)). Чтобы применить теорему 4 , мы должны проверить, что $F(v ; S)$ удовлетворяет ее условию. Для этого докажем следующие две леммы.

ЛЕмма 1. Отображение $F(v ; S)$ - гладкое отображение из пространства $H^{2}\left(\mathbb{R}^{2}\right) \times \mathbb{C}^{N}$ в $L^{2}\left(\mathbb{R}^{2}\right)$.

ДокАЗАтельство. Запишем $F$ в следующем виде:

$$
F(v ; S)=-\Delta v+\frac{|p(S)|^{2}}{1+|p(S)|^{2}}\left(e^{v}-1\right)-\frac{1}{1+|p(S)|^{2}}+\Delta \ln \left(1+|p(S)|^{2}\right) .
$$

Чтобы доказать, что отображение $F$ гладкое, достаточно показать, что $e^{v}-1$ лежит в $L^{2}\left(\mathbb{R}^{2}\right)$ для $v \in H^{2}\left(\mathbb{R}^{2}\right)$ и отображение $v \mapsto e^{v}-1$ - гладкое отображение из $H^{2}$ в $L^{2}$. Но согласно теореме вложения Соболева имеется непрерывное вложение пространства $H^{2}\left(\mathbb{R}^{2}\right)$ в пространство $\mathrm{BC}\left(\mathbb{R}^{2}\right)$ ограниченных непрерывных функций на $\mathbb{R}^{2}$, снабженное нормой $\|f\|_{\mathrm{BC}}=\sup _{x, y}|f(x, y)|$. Пользуясь этим, несложно доказать оценку

$$
\left\|v^{m}\right\|_{L^{2}} \leqslant C^{m-1}\|v\|_{L^{2}}\|v\|_{H^{2}}^{m-1},
$$

из которой и вытекает требуемое утверждение.

ЛЕмма 2. Обозначим через $w=w(S)$ единственное решение уравнения (10), соответствующее единственному решению $v$ уравнения (9). Это и есть неявная функиия, задаваемая уравнением $F(w(S) ; S)=0$. Тогда оператор $h \mapsto F_{v}^{\prime}(w(S) ; S) h$ $\left(\right.$ из $H^{2}\left(\mathbb{R}^{2}\right)$ в $L^{2}\left(\mathbb{R}^{2}\right)$ ) обратим для всякого $S \in \mathbb{C}^{N}$.

ДокАЗАТЕЛЬСтво. Для производной отображения $F$ по $v$ верна формула

$$
F_{v}^{\prime} h=-\Delta h+\frac{|p(S)|^{2}}{1+|p(S)|^{2}} e^{v} h
$$


Если $v=w(S)$, то коэффициент при $h$ имеет вид

$$
\begin{aligned}
& \frac{|p(S)|^{2}}{1+|p(S)|^{2}} e^{w(S)}= \\
& \quad=\frac{|p(S)|^{2}}{1+|p(S)|^{2}} \exp \left(v(S)-\sum_{k=1}^{N} \ln \left(\mu+\left|z-Z_{k}\right|^{2}\right)+\ln \left(1+|p(S)|^{2}\right)\right)= \\
& \quad=|p(S)|^{2} \exp \left(v(S)-\sum_{k=1}^{N} \ln \left(\mu+\left|z-Z_{k}\right|^{2}\right)\right)=|\Phi(S)|^{2} .
\end{aligned}
$$

Поэтому нам нужно доказать, что оператор $F_{v}^{\prime} h=A h:=-\Delta h+|\Phi|^{2} h$ имеет ограниченный обратный. Мы знаем, что $|\Phi|^{2}$ - гладкая функция на плоскости, $0 \leqslant|\Phi|^{2} \leqslant 1$ и $|\Phi| \rightarrow 1$ при $|z| \rightarrow \infty$.

Сначала покажем, что обратим оператор $B: H^{2}\left(\mathbb{R}^{2}\right) \rightarrow L^{2}\left(\mathbb{R}^{2}\right)$ вида $B h=-\Delta h+$ $s(x, y) h$, где $s(x, y)$ - гладкая функция, $\delta \leqslant s(x, y) \leqslant 1$ для некоторого $\delta>0$. Перепишем $B$ в виде $B h=-\Delta h+h+\psi(x, y) h$, где $\psi(x, y)=1-s(x, y)$. Заметим, что $\psi-$ гладкая функция, $0 \leqslant \psi(x, y) \leqslant 1-\delta$.

Используя преобразование Фурье

$$
\hat{u}=\mathcal{F}(u)=\frac{1}{2 \pi} \int_{\mathbb{R}^{2}} e^{-i\left(x \xi_{1}+y \xi_{2}\right)} u\left(\xi_{1}, \xi_{2}\right) d \xi_{1} d \xi_{2},
$$

мы можем показать, что

$$
\left\|\left(1+x^{2}+y^{2}\right) \hat{h}\right\|_{L^{2}} \leqslant \text { const } \cdot\|B h\|_{L^{2}}
$$

откуда $\|B h\|_{L^{2}} \geqslant \gamma\|h\|_{H^{2}}$ для некоторого $\gamma>0$. Далее, заметим, что

$$
B h=-\Delta h+h+\psi(x, y) h=B_{1} h+B_{2} h,
$$

где $B_{1} h=-\Delta h+h, B_{2} h=\psi(x, y) h$. Оператор $B_{1}$ обратим, поскольку

$$
B_{1}^{-1} h=\mathcal{F}^{-1}\left(\frac{\mathcal{F} h}{1+x^{2}+y^{2}}\right),
$$

поэтому $B_{1}$ сюръективен. Далее, $\left\|B_{1} h\right\|_{L^{2}}=\left\|\left(1+x^{2}+y^{2}\right) \hat{h}\right\|_{L^{2}} \geqslant\|\hat{h}\|_{L^{2}}=\|h\|_{L^{2}}$, a $\left\|B_{2} h\right\|_{L^{2}} \leqslant(1-\delta)\|h\|_{L^{2}}$. Из этого следует, что $B$ также сюръективен, а значит, $B$ обратим.

Чтобы доказать, что оператор $A=-\Delta+|\Phi|^{2}$ обратим, мы приблизим его обратимым оператором $A_{\epsilon}$, определяемым функцией $\epsilon$ со следующими свойствами: $\epsilon(x, y)-$ гладкая функция, $\epsilon(x, y) \geqslant 0$, она удовлетворяет неравенству $\delta \leqslant|\Phi|^{2}+\epsilon \leqslant 1$ для некоторого $\delta>0$ и обращается в нуль вне некоторого круга $B_{R}=B(0, R)$. Пусть $A_{\epsilon} h:=-\Delta h+\left(|\Phi|^{2}+\epsilon\right) h$ (этот оператор также отображает $H^{2}\left(\mathbb{R}^{2}\right)$ в $L^{2}\left(\mathbb{R}^{2}\right)$ ). Как уже доказано, оператор $A_{\epsilon}$ обратим и $A_{\epsilon} h=A h+\epsilon h$, поэтому $h=A_{\epsilon}^{-1} A h+A_{\epsilon}^{-1}(\epsilon h)$, откуда $A_{\epsilon}^{-1} A h=h-A_{\epsilon}^{-1}(\epsilon h)=\left(I-K_{\epsilon}\right) h$, где $I$ - тождественный оператор, $K_{\epsilon} h=$ $A_{\epsilon}^{-1}(\epsilon h)$. 
Очевидно, $K_{\epsilon}$ - компактный оператор, так как компактно вложение $H^{2}\left(B_{R}\right) \hookrightarrow$ $L^{2}\left(B_{R}\right)$. Теперь докажем, что уравнение $\left(I-K_{\epsilon}\right) h=0$ имеет лишь тривиальное решение в $H^{2}\left(\mathbb{R}^{2}\right)$. В самом деле, если $\left(I-K_{\epsilon}\right) h=0$, то $A h=A_{\epsilon}\left(I-K_{\epsilon}\right) h=0$, и, значит, $-\Delta h+|\Phi|^{2} h=0$. Умножая левую часть этого равенства на $h$ и интегрируя по частям, получаем

$$
(\nabla h, \nabla h)_{L^{2}}+\left(|\Phi|^{2} h, h\right)_{L^{2}}=0 .
$$

Но это значит, что $h=0$, так как $|\Phi| \rightarrow 1$ при $|z| \rightarrow \infty$. Из теоремы Фредгольма следует, что $I-K_{\epsilon}$ обратим, а тогда и оператор $A=A_{\epsilon}\left(I-K_{\epsilon}\right)$ также обратим.

Доказав эти леммы, мы можем теперь применить теорему о неявной функции и получить, что функция $w$ гладко зависит от $S_{1}, \ldots, S_{N}$ в $H^{2}\left(\mathbb{R}^{2}\right)$. Но $H^{2}$-норма мажорирует равномерную норму, а значит, $w$ гладко зависит от $S_{1}, \ldots, S_{N}$ (в терминах п. 1 теоремы 2). Тогда и функции $A_{1}, A_{2}, \Phi$, которые задаются формулами

$$
\begin{aligned}
& A_{1}=\partial_{2} \ln f=\frac{1}{2}\left(\partial_{2} w-\partial_{2} \ln \left(1+|p|^{2}\right)\right), \\
& A_{2}=-\partial_{1} \ln f=\frac{1}{2}\left(-\partial_{1} w+\partial_{1} \ln \left(1+|p|^{2}\right)\right), \\
& \Phi=p e^{\left(w-\ln \left(1+|p|^{2}\right)\right) / 2}=\frac{p}{\sqrt{1+|p|^{2}}} e^{w / 2},
\end{aligned}
$$

также гладко зависят от $S_{1}, \ldots, S_{N}$ (в терминах п. 1 теоремы 2).

Определение функций $\chi_{j, k}$. Подставляя выражения (6) в условие ортогональности, мы получим уравнение на функцию $\chi_{j, k}$ :

$$
-\Delta \chi_{j, k}+|\Phi|^{2} \chi_{j, k}=|\Phi|^{2} \frac{\partial \vartheta}{\partial S_{j, k}}
$$

где $\vartheta=\arg \Phi$.

Правая часть этого уравнения не лежит в $L^{2}$. Однако мы все же сможем воспользоваться обратимостью оператора $-\Delta+|\Phi|^{2}$, изменив правую часть вдали от нулей $\Phi$.

Пусть $\xi(r) \geqslant 0$ - гладкая функция от $r, r \geqslant 0$, со следующими свойствами: $\xi(r)=1$ при $0 \leqslant r \leqslant 1, \xi(r)$ убывает при $1 \leqslant r \leqslant 2$ и обращается в нуль при $r \geqslant 2$. Выберем гладкую функцию $R=R(S)$ так, чтобы круг $\{|z|<R\}$ содержал все нули многочлена $p(z)=z^{N}+S_{1} z^{N-1}+\cdots+S_{N-1} z+S_{N}$. Например, можно взять

$$
R(S)=R\left(S_{1}, \ldots, S_{N}\right)=\sqrt{1+\left|S_{1}\right|^{2}}+\sqrt[4]{1+\left|S_{2}\right|^{2}}+\cdots+\sqrt[2 N]{1+\left|S_{N}\right|^{2}} .
$$

Пусть $\rho(z ; S):=\xi(|z| / R(S))$. Тогда $\rho$ - гладкая функция с компактным носителем (по $z$ ), тождественно равная 1 в круге, содержащем все нули $\Phi$.

Предположим, что $\chi_{j, k}$ - гладкое решение (12). Рассмотрим функцию

$$
v_{j, k}:=\chi_{j, k}+(1-\rho) \frac{\partial \vartheta}{\partial S_{j, k}} .
$$

Эта функция удовлетворяет уравнению

$$
-\Delta v_{j, k}+|\Phi|^{2} v_{j, k}=-\rho|\Phi|^{2} \frac{\partial \vartheta}{\partial S_{j, k}}-\Delta\left((1-\rho) \frac{\partial \vartheta}{\partial S_{j, k}}\right) .
$$


В правой части этого уравнения стоит гладкая финитная функция. Поскольку оператор $-\Delta+|\Phi|^{2}$ обратим, оно имеет единственное решение в $H^{2}\left(\mathbb{R}^{2}\right)$, которое является гладким в силу известных теорем о регулярности решений эллиптических уравнений. Таким образом, вместо того чтобы решать уравнение (12) для $\chi_{j, k}$, мы решаем уравнение $(14)$ для $v_{j, k}$ и находим $\chi_{j, k}$ из соотношения (13).

Чтобы доказать, что $v_{j, k}$ гладко зависят от $S=\left(S_{1}, \ldots, S_{N}\right)$, мы опять используем теорему о неявной функции, применяя ее к отображению

$$
\widetilde{F}(v ; S):=-\Delta v+|\Phi(S)|^{2} v+\rho(S)|\Phi(S)|^{2} \frac{\partial \vartheta(S)}{\partial S_{j, k}}+\Delta\left((1-\rho(S)) \frac{\partial \vartheta(S)}{\partial S_{j, k}}\right) .
$$

Заметим, что $\widetilde{F}: H^{2} \times \mathbb{C}^{N} \rightarrow L^{2}$ есть гладкое отображение, а его производная по $v-$ обратимый оператор $-\Delta+|\Phi|^{2}$.

Теперь мы полагаем $\chi_{j, k}=v_{j, k}-(1-\rho) \partial \vartheta / \partial S_{j, k}$ и определяем $n_{j, k}$ выражением (6). Тогда $n_{j, k} \in\left(H^{1}\right)^{4}$, а отображения $\left(S_{1}, \ldots, S_{N}\right) \mapsto n_{j, k}\left(S_{1}, \ldots, S_{N}\right)$, рассматриваемые как отображения из $\mathbb{C}^{N}$ в $\left(L^{2}\right)^{4},-$ гладкие.

Линейная независимость $n_{j, k}$. Пусть $p(z)=p_{1}(z)+i p_{2}(z)$. Обозначим $\widetilde{w}:=2 \ln f$. Тогда

$$
\begin{aligned}
& n_{j, k}^{(1)}=\frac{\partial \Phi_{1}}{\partial S_{j, k}}-\Phi_{2} \chi_{j, k}=e^{\widetilde{w} / 2}\left(\frac{\partial p_{1}}{\partial S_{j, k}}+\frac{p_{1}}{2} \frac{\partial \widetilde{w}}{\partial S_{j, k}}-p_{2} \chi_{j, k}\right), \\
& n_{j, k}^{(2)}=\frac{\partial \Phi_{2}}{\partial S_{j, k}}+\Phi_{1} \chi_{j, k}=e^{\widetilde{w} / 2}\left(\frac{\partial p_{2}}{\partial S_{j, k}}+\frac{p_{2}}{2} \frac{\partial \widetilde{w}}{\partial S_{j, k}}+p_{1} \chi_{j, k}\right) .
\end{aligned}
$$

Предположим, что

$$
\sum_{j, k} c_{j, k} n_{j, k}^{(1)}=\sum_{j, k} c_{j, k} n_{j, k}^{(2)}=0
$$

для некоторых вещественных чисел $c_{j, k}$. Тогда

$$
\begin{aligned}
& \sum_{j, k} c_{j, k}\left(\frac{\partial p_{1}}{\partial S_{j, k}}+\frac{1}{2} p_{1} \frac{\partial \widetilde{w}}{\partial S_{j, k}}-p_{2} \chi_{j, k}\right)=0, \\
& \sum_{j, k} c_{j, k}\left(\frac{\partial p_{2}}{\partial S_{j, k}}+\frac{1}{2} p_{2} \frac{\partial \widetilde{w}}{\partial S_{j, k}}+p_{1} \chi_{j, k}\right)=0 .
\end{aligned}
$$

Умножая первое уравнение на $2 p_{1}$, второе на $2 p_{2}$ и складывая их, получаем

$$
\sum_{j, k} c_{j, k}\left(2 p_{1} \frac{\partial p_{1}}{\partial S_{j, k}}+2 p_{2} \frac{\partial p_{2}}{\partial S_{j, k}}+\left(p_{1}^{2}+p_{2}^{2}\right) \frac{\partial \widetilde{w}}{\partial S_{j, k}}\right)=0
$$

что можно записать как

$$
\sum_{j, k} c_{j, k}\left(\frac{\partial|p|^{2}}{\partial S_{j, k}}+|p|^{2} \frac{\partial \widetilde{w}}{\partial S_{j, k}}\right)=0
$$

Функция $\widetilde{w}$ удовлетворяет уравнению $\Delta \widetilde{w}=|p|^{2} e^{\widetilde{w}}-1$. Дифференцируя его по параметрам $S_{j, k}$, мы получаем

$$
\Delta \frac{\partial \widetilde{w}}{\partial S_{j, k}}=e^{\widetilde{w}}\left(\frac{\partial|p|^{2}}{\partial S_{j, k}}+|p|^{2} \frac{\partial \widetilde{w}}{\partial S_{j, k}}\right) .
$$


С помощью (16) из последнего уравнения мы можем заключить, что

$$
\sum_{j, k} c_{j, k} \Delta \frac{\partial \widetilde{w}}{\partial S_{j, k}}=e^{\widetilde{w}} \sum_{j, k} c_{j, k}\left(\frac{\partial|p|^{2}}{\partial S_{j, k}}+|p|^{2} \frac{\partial \widetilde{w}}{\partial S_{j, k}}\right)=0 .
$$

Другими словами, функция $\sum_{j, k} c_{j, k} \partial \widetilde{w} / \partial S_{j, k}$ гармонична. Однако частные производные

$$
\frac{\partial \widetilde{w}}{\partial S_{j, k}}=\frac{\partial w}{\partial S_{j, k}}-\frac{\partial \ln \left(1+|p|^{2}\right)}{\partial S_{j, k}}=\frac{\partial w}{\partial S_{j, k}}-\frac{\partial|p|^{2}}{\partial S_{j, k}} \frac{1}{1+|p|^{2}}
$$

стремятся к нулю при $|z| \rightarrow \infty$. Из теоремы Лиувилля вытекает, что

$$
\sum_{j, k} c_{j, k} \frac{\partial \widetilde{w}}{\partial S_{j, k}} \equiv 0
$$

Тогда из уравнения (16) мы получаем, что

$$
\sum_{j, k} c_{j, k} \frac{\partial|p|^{2}}{\partial S_{j, k}} \equiv 0
$$

но это возможно лишь тогда, когда все коэффициенты $c_{j, k}$ нулевые.

Благодарности. Работа частично поддержана РФФИ (гранты № 05-01-00498, 05-01-00370) и Программой поддержки ведущих научных школ (грант № НШ$6705.2006 .1)$.

\section{Список литературы}

[1] А. Г. Сергеев, С. В. Чечин, ТМФ, 85:3 (1990), 397-411.

[2] T. M. Samols, Comm. Math. Phys., 145:1 (1992), 149-179.

[3] P. J. Ruback, Nucl. Phys. B, 296:3 (1988), 669-678.

[4] F. Abdelwahid, J. Burzlaff, J. Math. Phys., 35:9 (1994), 4651-4660.

[5] A. Jaffe, C. Taubes, Vortices and Monopoles, Prog. Phys., 2, Birkhäuser, Boston, 1980.

[6] D. Stuart, Comm. Math. Phys., 159:1 (1994), 51-91.

[7] N. S. Manton, Phys. Lett. B, 110:1 (1982), 54-56.

[8] R. MacKenzie, Phys. Lett. B, 352 (1995), 96-98; arXiv: hep-th/9503044.

[9] K. Arthur, J. Burzlaff, Lett. Math. Phys., 36:3 (1996), 311-318; arXiv: hep-th/9503010.

Поступила в редакцию 25.12.2006, после доработки 3.05.2007 